\title{
Few-Cycle Optical Pulses in Thin Film of Topological Insulator with Taking into Account the Nonlinearity of Medium
}

\author{
N.N. Konobeeva ${ }^{1, *}$, M.B. Belonenko ${ }^{2}$, A.V. Zhukov ${ }^{3}$ \\ ${ }^{1}$ Volgograd State University, Volgograd, 400062, Russia \\ ${ }^{2}$ Volgograd Institute of Business, Volgograd, 400048, Russia \\ ${ }^{3}$ Singapore University of Technology and Design, 20 Dover Drive, Singapore \\ *Corresponding Author: yana_nn@inbox.ru
}

Copyright (C) 2013 Horizon Research Publishing All rights reserved.

\begin{abstract}
The propagation of few-cycle optical pulse in a thin film of topological insulator with allowance for the proper nonlinearity of the medium is considered. We considered a simple model of medium susceptibility. And approximation where there are no transitions between the valence band and the conduction band was taken into account. The dependence of the pulse on the nonlinearity constant of the medium is revealed. The evolution of the electromagnetic pulse is studied.
\end{abstract}

Keywords Optical Pulse, Topological Insulator, Nonlinearity

\section{Introduction}

Now, the field associated with topological insulators study is became more and more attractive for researches. The question of the interaction of topological insulators with intense external electromagnetic field (for example, with optical pulse field) is not ignored.

At the same time, it is worth noting the propagation of few-cycle pulses in optical media without destroying the substance is possible at large intensities, which, in turn, have made it possible to observe nonlinear phenomena that are rather rare in a field of long pulses [1]. The problem of higher order harmonics generation was investigated in [2].

Few-cycle pulses mean pulses, which consist of several field oscillations [3-5]. In [6] carbon nanotubes were selected as a medium for pulses and a practically important case (used in the analysis of pulse propagation in the waveguides structure), which takes into account own dispersion medium was consider. This work considers the dynamics of few-cycle pulses that propagate in thin film of topological insulators capable of displaying nonlinear properties in dispersive nonmagnetic media. It can be very important for practical applications [7-9].

\section{Methods and Results}

\subsection{Basic Equations}

We consider a thin topological insulator film, whose Hamiltonian will be taken in a long-wave approximation [10]:

$$
\begin{aligned}
& H=\left(p_{x}^{2}+p_{y}^{2}\right) / 2 m+v_{f}\left(p_{x} \sigma_{y}-p_{y} \sigma_{x}\right)+\frac{\lambda}{2}\left(p_{+}^{3}+p_{-}^{3}\right) \sigma_{z} \\
& p_{ \pm}=p_{x} \pm i p_{y}
\end{aligned}
$$

$p_{x}, p_{y}$ are the electron-momentum components, $m$ is the electron effective mass, $\sigma_{x}, \sigma_{y}$ are the spin matrices, $v_{f}$ is the Fermi velocity, $\lambda$ is the parameter related to the hexagonal distortions.

Hamiltonian (1) can be readily diagonalized, which specifies the electron spectrum:

$$
\varepsilon\left(p_{x}, p_{y}\right)=\frac{\left(p_{x}^{2}+p_{y}^{2}\right)}{2 m}+\sqrt{\lambda^{2} p_{x}^{2}\left(p_{x}^{2}-3 p_{y}^{2}\right)+v_{f}^{2}\left(p_{x}^{2}+p_{y}^{2}\right)}
$$

According to quantum-mechanical rules, in the presence of the electric field, (which is for certainty directed along the $\mathrm{x}$ axis and further assumed to obey the calibration: $\boldsymbol{E}=-\partial \boldsymbol{A} / \mathrm{c} \partial \mathrm{t}$, the momentum must be replaced by the generalized momentum: $p \rightarrow p-e A / c$. In this case, Hamiltonian (1) can be rewritten in the following form:

$$
H=\sum_{p \sigma} \varepsilon\left(p-\frac{e}{c} A(t)\right) a_{p \sigma}^{+} a_{p \sigma}
$$

where $a_{p \sigma}^{+}, a_{p \sigma}$ are the creation and annihilation operators of electrons. 
The Maxwell's equations with the inclusion of the dielectric and magnetic properties of the topological insulator can be written in the quasi one-dimensional approximation in the form [11]:

$$
\frac{\partial^{2} \boldsymbol{E}}{\partial z^{2}}-\frac{1}{c^{2}} \frac{\partial^{2} \boldsymbol{E}}{\partial t^{2}}+\frac{4 \pi}{c} \frac{\partial \boldsymbol{j}}{\partial t}=\frac{4 \pi}{c^{2}} \frac{\partial^{2} \boldsymbol{P}}{\partial t^{2}}
$$

Here, we neglect the diffraction spread of the laser beam across the propagation direction. Here $\boldsymbol{P}=\alpha \boldsymbol{E}+\beta|\boldsymbol{E}|^{2} \boldsymbol{E}$ is the polarization response of the medium. Here, we took the simplest model for the nonlinearity of the medium in which the polarization vector is assumed to be parallel to vector $\boldsymbol{E}$. $\mathrm{Eq}(4)$ is generalized for the case of a nonlinear medium by the equation of the form:

$$
\frac{\partial^{2} \boldsymbol{A}}{\partial z^{2}}-\frac{1}{c^{2}}(1+4 \pi \alpha) \frac{\partial^{2} \boldsymbol{A}}{\partial t^{2}}-\frac{12 \pi \beta}{c^{4}} \frac{\partial^{2} \boldsymbol{A}}{\partial t^{2}}\left(\frac{\partial \boldsymbol{A}}{\partial t}\right)^{2}+\frac{4 \pi}{c} \boldsymbol{j}=0
$$

Vector potential $\boldsymbol{A}$ is assumed to have the form $\boldsymbol{A}=(0, A(\mathrm{z}, t), 0)$.

Let us write the standard expression for the current density:

$$
j_{0}=e \sum_{p s} v_{s}\left(p-\frac{e}{c} A(t)\right)\left\langle a_{p s}^{+} a_{p s}\right\rangle
$$

where $v_{\mathrm{s}}(p)=\partial \varepsilon_{\mathrm{s}}(p) / \partial p$, and the angular brackets denote averaging with non-equilibrium density matrix: $\rho(\mathrm{t})$ $<B>=S p(B(0) \rho(\mathrm{t}))$. Taking into account that $\left[a_{p s}^{+} a_{p s}, H\right]=0$ we immediately obtain from the equations of motion for the density matrix the equality: $\left\langle a_{p s}^{+} a_{p s}\right\rangle=\left\langle a_{p s}^{+} a_{p s}\right\rangle_{0}$, where $\langle B\rangle_{0}=S p(B(0) \rho(0))$.

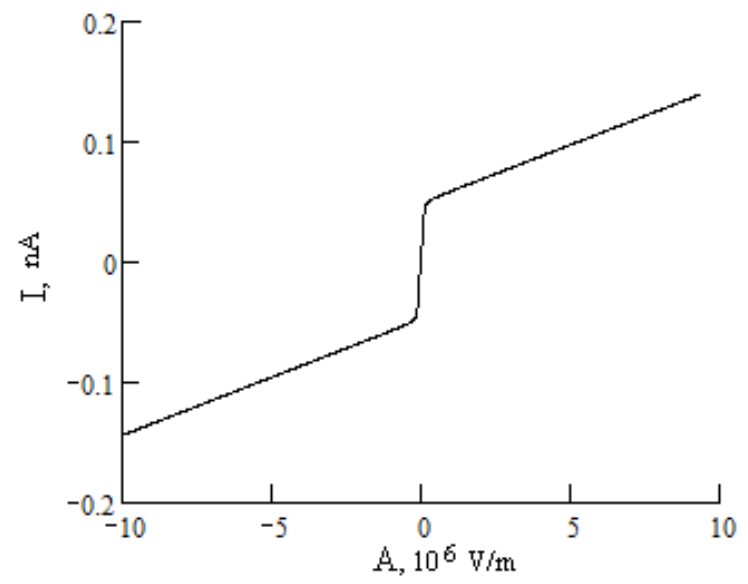

Figure 1. Current versus the magnitude of the vector potential

Below we will consider low temperatures, when only a narrow region of the momentum space near the Fermi level contributes to sum (integral) (6). Accordingly, Eq. (6) reads as:

$$
j=e \int_{-\Delta-\Delta}^{\Delta} \int_{-\Delta}^{\Delta} d p_{x} d p_{y} v_{y}\left(p-\frac{e}{c} A(x, t)\right)
$$

The integration area $\Delta$ in the momentum space in Eq. (7) can be found from the conservation of particles:

$$
\int_{-\Delta-\Delta}^{\Delta} \int_{x}^{\Delta} d p_{x} d p_{y}=\int_{-\Delta-\Delta}^{\Delta} \int_{-\Delta}^{\Delta} d p_{x} d p_{y}\left\langle a_{p x, p y}^{+} a_{p x, p y}\right\rangle
$$

the integration domain is the first Brillouin zone.

In this case, the equation for the propagation of an extremely short pulse can be written as:

$$
\frac{\partial^{2} \boldsymbol{A}}{\partial z^{2}}-\frac{1}{c^{2}}(1+4 \pi \alpha) \frac{\partial^{2} \boldsymbol{A}}{\partial t^{2}}-\frac{12 \pi \beta}{c^{4}} \frac{\partial^{2} \boldsymbol{A}}{\partial t^{2}}\left(\frac{\partial \boldsymbol{A}}{\partial t}\right)^{2}+\frac{4 \pi}{c} \Phi(\boldsymbol{A})=0
$$

here $\Phi(\boldsymbol{A})$ is given by integration in (7).

Typical dependence of the nonlinearity entering Eq. (7) on the magnitude of the vector potential A is shown in (fig. 1).

\subsection{Results and Discussions}

Equation (8) was solved numerically with the use of an explicit five-point finite difference scheme [12]. The time and space steps were found from the standard stability conditions. The initial condition was taken in the form:

$$
\begin{aligned}
& A(0, t)=Q \cdot \exp \left(-v^{2} t^{2} / g^{2} \gamma\right) \\
& \frac{d A(0, t)}{d z}=\frac{2 Q v t}{g \gamma} \cdot \exp \left(-v^{2} t^{2} / g^{2} \gamma\right) \\
& \gamma=\left(1-v^{2} / c^{2}\right)^{1 / 2}
\end{aligned}
$$

where $Q$ is the amplitude of pulse, $v$ is the pulse velocity, $g$ is the pulse width (space localization of pulse), $c$ is the light speed in a vacuum.

The evolution of the electric field during its propagation in the sample is shown in (fig. 2).

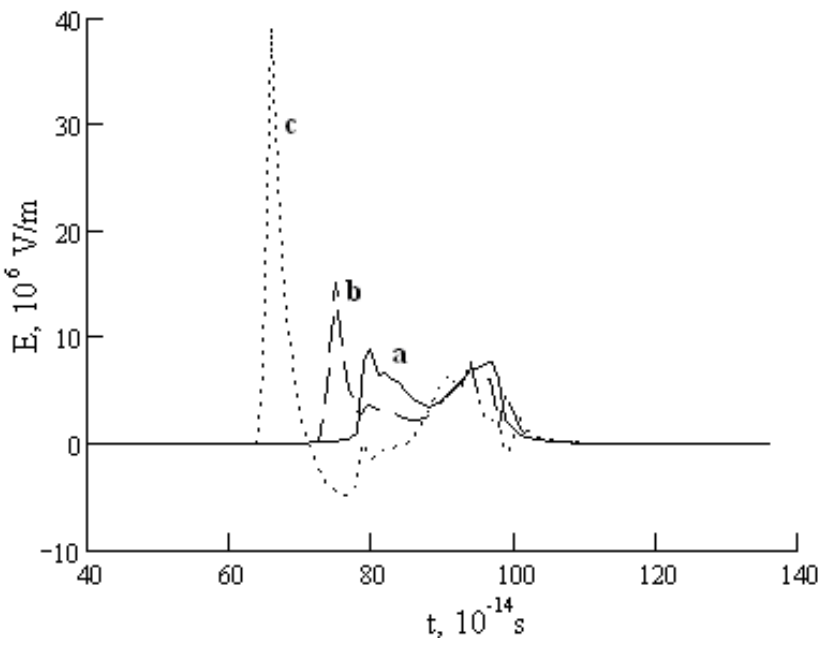

Figure 2. Time dependence of the electric field on time at various space points (a) $\mathrm{z}=10^{-5} \mathrm{~m}$, (b) $1.5 \times 10^{-5} \mathrm{~m}$, and (c) $2 \times 10^{-5} \mathrm{~m}$.

According to the present results, we have increasing of the delayed pulse amplitude is connected with the influence of the proper nonlinearity of the medium. It is seen from fig. 2 that the pulse is split into two pulses, this splitting increasing with an increase in the distance traversed. With an increase 
in the traversed distance, the amplitude of the delayed pulse increases. Such behavior is associated with a balance of dispersion and nonlinearity of medium.

For the main pulse there is "a tail" (fig.3), which consists of two smaller amplitude pulses. With an increase in linear constant $\alpha$, the momentum remains more long-lived.

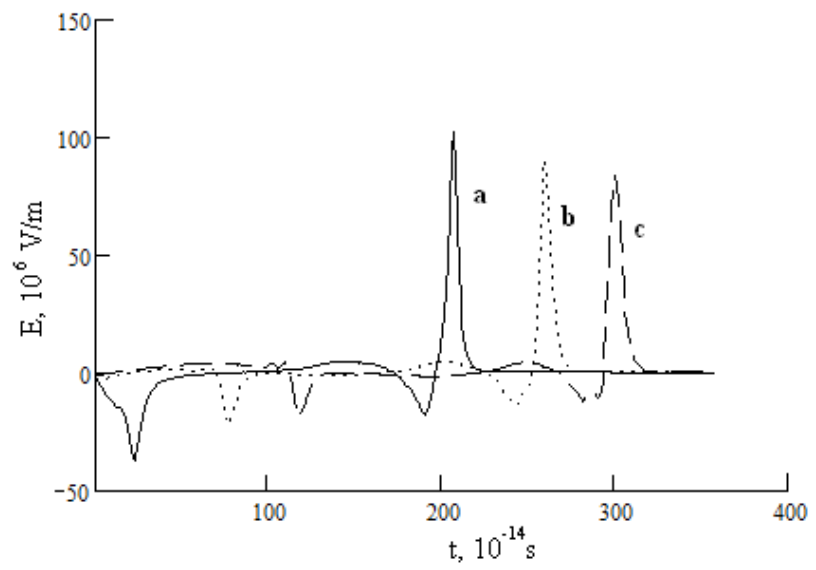

Figure 3. Time dependence of the electric field on time at various nonlinearity parameters (a) $\alpha=0.5, \beta=0.001$; (b) $\alpha=0.1, \beta=0.001$; (c) $\alpha=1.5$, $\beta=0.001$

The nonlinearity coefficient $\beta$ do not much affect the duration of an optical pulse (fig.4), but only determines the peaks number of propagating pulse in the media. "A tail" after the pulse is not observed.

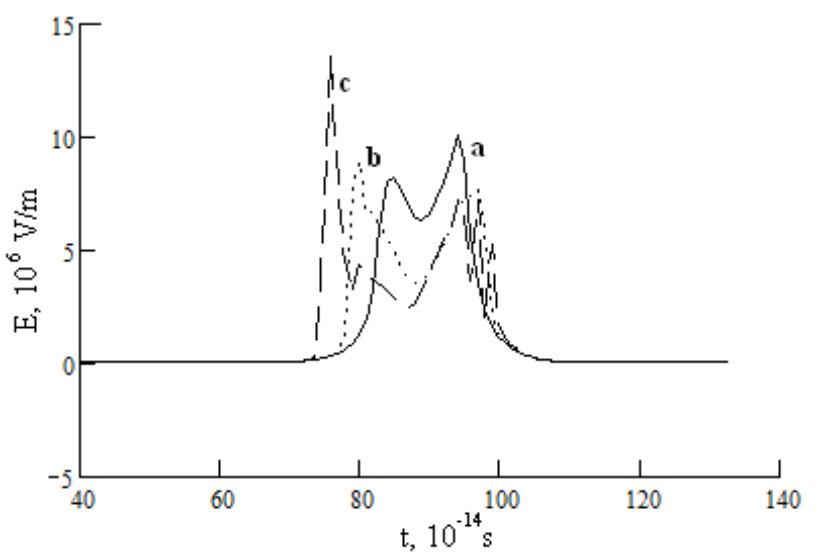

Figure 4. Time dependence of the electric field on time at various nonlinearity parameters (a) $\alpha=0.5, \beta=-0.1$; (b) $\alpha=0.5, \beta=-0.5$; (c) $\alpha=0.5$, $\beta=-1.0$

Since $\beta<0$, the peak of the pulse decreases its amplitude due to the nonlinearity and two peaks are even formed. The peaks evolve further with allowance for the nonlinearity caused by the nonlinearity of the current in the topological insulator. With an increase in nonlinearity constant $\beta$, the retarding peak increases faster than the leading one, which proves the aforementioned mechanism of the pulse shape formation.

In the end of this paper, we state the main conclusions of this work. Account of the nonlinearity affects the propagation few-cycle optical pulses in topological insulators, which manifests itself in the changing shape, pulse amplitude and in the process of "a tail" formation.

\section{Conclusion}

The key conclusions from this work are below.

1. An effective equation was obtained for the dynamics of few-cycle optical pulses in thin film of topological insulator with allowance for the proper nonlinearity of the medium.

2. It was shown that the propagation of ultimately few-cycle optical pulses in thin film of topological insulator with allowance for the nonlinearity of the medium is determined by both the polarization response constants.

The pulse behavior is associated with a balance of dispersion and nonlinearity of the medium.

3. Such pulses in the femtosecond lasers could be used in spectroscopy, in the delay lines.

\section{Acknowledgements}

This work was supported by the Russian Foundation for Basic Research (project no. 12-02-31654).

\section{REFERENCES}

[1] D. Von der Linde, H. Schuler. Breakdown threshold and plasma formation in femtosecond laser-solid interaction, Journal of the Optical Society of America B, Vol. 13, No.1, 216-222, 1999.

[2] M.B. Belonenko. S.Yu. Glazov. N.E. Mesheryakova, Influence of an alternating electric field on the conductivity of single-wall semiconductor carbon nanotubes, Semiconductors, Vo.44, No. 9, 1248-1253, 2010.

[3] A. Baltuska, Z. Wei, M. S. Pshenichnikov, D. A. Wiersma, Optical pulse compression to $5 \mathrm{fs}$ at a $1-\mathrm{MHz}$ repetition rate, Optical Letters, Vol. 22. No.2, 102-104, 1997.

[4] M. Nisoli, S. De Silvestri, O. Svelto, R. Szipocs, K. Ferencz, Ch. Spielmann, S. Sartania, F. Krausz, Compression of high-energy laser below 5 fs, Optical Letters, Vol. 22, No.8, 522-524, 1997.

[5] Th. Brabec, F. Krausz, Intense few-cycle laser fields: Frontiers of nonlinear optics, Reviews of Modern Physics, Vol. 72, No. 2, 545-591, 2000.

[6] N. N. Yanyushkina, M. B. Belonenko, N. G. Lebedev, Ultimately short optical pulses in carbon nanotubes in dispersive nonmagnetic dielectric media, Optics and spectroscopy, Vol. 111, No.1, 92-97, 2011.

[7] D. Hsieh, Y. Xia, D. Qian, L. Wray, J. H. Dil, F. Meier, J. Osterwalder, L. Patthey, N. P. Checkelsky, N. P. Ong, A. V. Fedorov, H. Lin, A. Bansil, D. Grauer, Y. S. Hor, R. G. Cava, M. Z. Hasan, A tunable topological insulator in the spin 
helical Dirac transport regime, Nature, Vol. 460, 1101-1105, 2009.

[8] Ya. Tserkovnyak, D. Loss, Thin-film magnetization dynamics on the surface of a topological insulator, Physical Review Letters, Vol. 108, No.18, 187201, 2012.

[9] R. W. Reinthaler, E. M. Hankiewicz, Interplay of bulk and edge states in transport oft wo-dimensional topological insulators, Physical Review B, Vol. 85, 165450, 2012.
[10] H. Lin, R. S. Markiewicz, L. A. Wray, L. Fu, M. Z. Hasan, A. Bansil, Single-Dirac-Cone topological surface states in TIBiSe2 class of Topological Insulators, Physical Review Letters, Vol. 105, 036404, 2010.

[11] M. B. Vinogradova., O. V. Rudenko, A. P. Sukhorukov. The Theory of Waves, Nauka, Moscow, 1990.

[12] N. S. Bahvalov. Calculation methods (analysis, algebra, ordinary differential equations), Nauka, Moscow, 1975. 